\title{
Statistical Mechanics of Two Dimensional Tilings
}

\author{
Forrest H. Kaatz \\ Mesalands Community College, 911 South Tenth Street, Tucumcari, NM 88401 \\ Electronic mail: fhkaatz@gmail.com
}

Ernesto Estrada

Institute of Complex Systems at Strathclyde, Department of Mathematics and Department of Physics, University of Strathclyde, Glasgow G1 1XH, UK

\author{
Adhemar Bultheel \\ Department of Computer Science, K.U.Leuven, Celestijnenlaan 200A, 3001 Heverlee, \\ Belgium
}

Noel Sharrock

Lochgardie Media, PO Box 67, Shepparton East, Victoria, 3631 Australia

\begin{abstract}
Reduced dimensionality in two dimensions is a topic of current interest. We use model systems to investigate the statistical mechanics of ideal networks. The tilings have possible applications such as the 2D locations of pore sites in nanoporous arrays (quantum dots), in the 2D hexagonal structure of graphene, and as adsorbates on quasicrystalline crystal surfaces. We calculate the statistical mechanics of these networks, such as the partition function, free energy, entropy, and enthalpy. The plots of these functions versus the number of links in the finite networks result in power law regression. We also determine the degree distribution, which is a combination of power law and rational function behavior. In the large-scale limit, the degree of these 2D networks approaches 3, 4, and 6, in agreement with the degree of the regular tilings. In comparison, a Penrose tiling has a degree also equal to about 4 .
\end{abstract}

Keywords: regular tilings; degree distribution; power laws; finite graph; statistical mechanics functions; Penrose tiling 


\section{Introduction}

The statistical mechanics of networks has received increased attention in recent years [1]. From a mathematical or physics perspective, some of the earliest networks investigated were random graphs [2]. Graph theory has quite a long history of over 50 years since the initial investigations on random graphs. Graphs need not be random, and in fact it is well known [3] that in two dimensions, it is possible to tile the plane with 3,4 , 6 , or even 5-fold symmetry if one allows for tiles of different shapes.

The Archimedean lattices describe methods of tiling the plane with geometric polygons [4]. For these, we require: (a) that the tiling be edge-to-edge; (b) that the tilings be regular polygons; and (c) that all vertices be of the same type [4]. This then results in 11 distinct tilings known as the Archimedean or Kepler tilings. The Schläfli symbols that describe the polygon type and the degeneracy type that meets at an equivalent vertex labels them appropriately. If we further require that the tilings be constructed with regular polygons that are monohedral, i.e., the same size and shape, we have the three regular tilings, $3^{6}, 4^{4}$, and $6^{3}$.

We also take a look at two-dimensional quasicrystalline or 'Penrose tilings' with five-fold symmetry. These arrays are created with two different rhombs, one thin and one thick, with the same edge length. We mention here that the five-fold symmetry seen in Fourier diffraction patterns [5], is a result of medium to long range order in the graph, which we confirm by finding the number of nearest neighbors $(\approx 4)$ in these plots.

The regular tilings may have applications in the real world, as the coordinates define the locations of pore sites in nanoporous arrays [6]. All three of the regular tilings have been fabricated by nanolithography into (non-ideal) porous arrays for the definition of nanosized material (dots or wires). Also, two-dimensional hexagonal networks of carbon have been made, in the form known as graphene [7]. The 2D nature of graphene is 
not ideal or perfectly flat, but when suspended in air has ripples on the order of $1 \mathrm{~nm}$. [8]. The 2D structure of Penrose tilings may be applicable to surface studies [9] of adsorbates on quasicrystalline single crystal surfaces. We have previously calculated some Euclidean distance matrices and the Wiener Indices of several graphs of nanoarrays [10]. Here our focus is on the statistical mechanics of the ideal finite networks.

Many of the fundamental characteristics and calculations for graphs can be achieved from the knowledge of its associated adjacency matrix. The adjacency matrix is constructed by numbering the vertices in the graph; if there is a link between two adjacent vertices, the corresponding matrix element is one. If there is no link, the matrix element is zero. If we define the matrix element to be the actual distance between the vertices, then we are using a Euclidean adjacency matrix [10].

The statistical mechanics approach to complex networks has been developed since it lends itself to reducing seemingly complex calculations on many elements into relatively simple analysis. There are a number of terms [1] that have been developed with this analysis to clarify the type of network under consideration. Thus 'random' graphs generally have a specified probability that two vertices are connected; 'clustering' refers to the number of links each vertex has in relation to the total number of links; 'small world' refers to the number of links separating two vertices along the shortest path between them, with a general consensus that an average of six over the whole graph defines the small world concept; 'degree distribution' describes the relationship between vertices and the number of links between them, in other words, the number of nearest neighbors. If the degree distribution has a power law behavior, it is said to be 'scale-free'. Using the statistical mechanics approach, one can calculate the usual relevant parameters such as: the partition function, free energy, entropy, and enthalpy, once one has determined the adjacency matrix [11]. In the present manuscript, we determine these 
functions for the regular tilings and a well defined 2D random graph. As an aside, we mention that the random graph does not correspond to a nanoporous array since it has a dense Fourier transform, but random networks are under active investigation as models of other systems [1].

\section{Experimental}

MATLAB, Excel, and Kaleidagraph are the software tools necessary to create and analyze the data. In the graphs we use, the Euclidean distance between vertices is calculated, and vertices are connected up to 1.15 times the minimum distance between every two vertices. This generates all the regular tilings with the appropriate nodes and links, without extraneous links occurring. The 2D random graph uses a uniform random distribution to assign 2D coordinates, and connects links between two vertices if the Euclidean distance is less than 1.15 times the smallest distance (any factor less than $\sqrt{2}$ will work). We generate finite networks of about 10, 100, 500, 1000, and 10,000 vertices and plot the results. The MATLAB code generates the plots of the graphs, the values of the statistical mechanics functions, and the average degree of vertices in the graph. The processing time goes as the fourth power of the number of vertices, with 10,000 vertices taking about 1900 seconds.

\section{Methods}

\subsection{Local Geometry}

The regular tilings can be generated by the equations:

$$
\mathbf{a}_{1}=a \hat{x} \quad \mathbf{a}_{2}=a \cos \left(\frac{2 \pi}{n}\right) \hat{x}+a \sin \left(\frac{2 \pi}{n}\right) \hat{y}
$$

where $\mathbf{a}_{1}$ and $\mathbf{a}_{2}$ are basis vectors for the sites or vertices, $a$ is the distance between sites, and $n$ is the symmetry of the lattice, 3,4 , or 6 . In addition, a hexagonal tiling is actually 
constructed from two interconnected equilateral triangles with a basis shift of the second triangle given by:

$$
b_{x}=a_{1 x}+a_{2 x}+a \cdot \frac{1}{2} \quad b_{y}=a_{2 y}+a \cdot \frac{\sqrt{3}}{6}
$$

where $a$ is again the distance between sites. The MATLAB code plots graphs from the adjacency matrix, which only includes connections from nearest neighbors. In Table 1, we list the first and second nearest neighbor distances for regular tilings. We see that the random graph could have used any factor up to $\sqrt{ } 2$, since that is the smallest value for the second nearest neighbor.

We also used Penrose tilings in this work. The two rhombs for generating these plots is shown in Figure 1. The 'bond length' is $a$, and is the same for both rhombs. It can be seen that in the narrow rhomb, the distance across vertices B and D is the nearest neighbor in these graphs. The distance from $\mathrm{Q}$ to $\mathrm{S}$ in the second rhomb is the third neighbor, so that the distance of interest are all second neighbors of length $a$. If we normalize to the smallest distance, then $a$ has length $\tau \approx 1.618$, the golden mean. In the MATLAB code, we create two adjacency matrices that cut off distances below and above the golden mean, to create a plot with the second neighbors. We calculate the number of neighbors for each site for a Penrose tiling of 12,079 vertices and plot the results in Figure 2. The maximum value is $L_{D}=7$, with 653 sites having this configuration, where $L_{D}$ is the local degree of a site. If we cut the $L_{D}=1,2$ coordinated sites as being border vertices of the graph, we get an average degree of $d_{\infty}>3.987$ (where $d_{\infty}$ is the average degree of vertices in the graph for a large array). A small number of the $L_{D}=3$ sites and above are also likely to be border vertices. In Figure 3, we show the graphs resulting from connecting a nominal 300 vertices with random, 3, 4, 6-fold symmetry, along with a Penrose tiling. These are shown as evidence that since the MATLAB plot is derived from 
the adjacency matrix, we can have confidence that the calculations proceed as designed, as the calculations depend on an accurate adjacency matrix.

\subsection{Statistical Mechanics}

The adjacency matrix has been described previously. Here we show how to calculate the statistical mechanics functions from the adjacency matrix $\mathbf{A}$ [11]. The partition function of a graph $G$ with 'inverse temperature' $\beta=1 /\left(k_{B} \mathrm{~T}\right)$, is defined as the trace of the following matrix:

$$
Z(G, \beta)=\operatorname{Tr}\left(e^{\beta \mathrm{A}}\right)
$$

where $Z$ is the partition function. We set $\beta=1$ in our calculations Note that $\beta$ can be considered the 'strength' of an interaction between two vertices [11], so that in principle one could create a more realistic model for 2D applications based on its' value.

In following with the statistical mechanics approach, we define the probability $p_{j}$ that the ensemble occupies a microstate $j$ to be:

$$
p_{j}=\frac{e^{\beta_{j}}}{Z(G, \beta)}
$$

where $\lambda_{j}$ is an eigenvalue of the adjacency matrix $\mathbf{A}$. The (Shannon) entropy [13] of the graphs can then be defined as follows:

$$
S(G, \beta)=-k_{\overline{\text { मे }}} \sum_{j}\left[p_{j}\left(\beta \lambda_{j}-\ln Z(G, \beta)\right)\right]
$$

or if it is rewritten:

$$
S(G, \beta)=-k_{B} \beta \sum_{j} \lambda_{j} p_{j}+k_{g} \ln (Z) \sum_{j} p_{j}
$$

where $\beta=1 /\left(k_{B} \mathrm{~T}\right)$, and $k_{B}$ is the Boltzman constant. We plot the entropy / link vs. the number of links in Fig. 4(a). The data is generated from finite networks of about 10, 100, 500,1000 , and 10,000 vertices linked together. (The lower limit of vertices changes from 
one graph to another.) We see that the triangular graph has the most number of links at 60,990, which corresponds to its 6-fold degree. Surprisingly, all the plots are similar, with an average power law fit of about $S / l=1.43 \cdot l^{-0.81}$.

We write the Helmholtz free energy $F(G)$ in terms of the enthalpy $H$ and entropy $S$ in the standard way as $F=H-T S$. This results in the free energy represented as the natural logarithm of the partition function:

$$
F(G, \beta)=-\frac{\ln Z(G, \beta)}{\beta}
$$

and the enthalpy written as follows:

$$
H(G, \beta)=-\frac{1}{Z(G, \beta)} \operatorname{Tr}\left(\mathbf{A} e^{\beta \mathrm{A}}\right)
$$

where $\mathbf{A}$ and $Z$ have the standard definitions. We plot the enthalpy/link and free energy/link in Figs. 4(b) and 4(c), respectively. The most notable feature of the enthalpy graph is that the random graph is steeper than the rest, and has perfect regression through the data points. The power-law fits of the enthalpy/link in the random graph is better than the other graphs, and seem to decrease from the fit with a large number of links. The free energy plots look similar to the entropy plots, with a similar power regression, but differing in sign.

We mentioned that scale-free networks typically have a power law fit to the degree distribution. In such cases, it is possible to get an explicit form for the degree distribution relationship [14]. In our case, we know that the large-scale limit for the regular tilings have degrees or nearest neighbors of 3, 4, and 6. In the MATLAB code we calculate the degree as the quotient of the number of links and the number of vertices in A, and plot the results in Fig. 5. We use an empirical fit of the form: 


$$
V\left(d_{n}\right)=a+\frac{b \cdot d^{c}}{\left(d_{u}-d_{\infty}\right)}
$$

where $V$ is the number of vertices, $a, b$, and $c$ are constants of the fit, $d_{\infty}$ is the average degree of vertices in the graph as the size of the graph gets large; either 3,4 , or 6 ; $d_{n}$ is the average degree of vertices in the graph for a specific size graph and $\mathrm{n}$ is the number of finite vertices in the graph. Note that $d_{n}$ and $V$ take on discrete values and thus $V$ is not a continuous function. The fit parameters are summarized in Table 2. These fits are optimized using the Curve Fitting Toolbox in MATLAB. We plot the number of vertices vs. the degree for the five different graphs. The curvature at low degree is due to the number of border vertices with fewer links as a fraction of the total number of vertices, and $d_{\infty}$ appears as a vertical asymptote in the rational function fits. We find that the Penrose tiling has a value of $d_{\infty} \approx 4$, less than the 5-fold symmetry from the long-range order. At low values of $d$, the only data point we can be confident about is the coordinate $(1,2)$ for the random graph, which is interpreted as two vertices having one nearest neighbor. For the other tilings, how the network grows will determine the degree and there is no unique configuration for small networks. From the plot we see that the Penrose tiling requires the largest number of vertices for the minimum features of the graph, which corresponds to the long-range character of the tiling. If we require that the graphs be 'compact', i.e. have a minimum number of border vertices, we feel confident that the regression fits are accurate.

\section{Conclusions}

In summary, we have determined the statistical mechanics functions and degree distribution for the regular tilings, a random graph, and a Penrose tiling. These model systems give us an idea of how the statistical mechanics of finite networks behave as they become larger. The statistical mechanics plots of the entropy, free energy, and enthalpy 
all have power law behavior, with good regression features. The degree distributions follow a combination of power law and rational function character, which explains the effect of border vertices and number of links in the graph. We estimate that a Penrose tiling with 5-fold symmetry has degree $d_{\infty} \approx 4$.

\section{Acknowledgments}

We thank the anonymous referees and the editors for comments resulting in corrections that clarified the manuscript. 


\section{References}

1. R. Albert, A.L. Barabasi, Statistical mechanics of complex networks, Rev. of Modern Physics, 74 47-97 (2002)

2. P. Erdos, A. Renyi, On the evolution of random graphs, Bull. Internat. Statist. Inst., 38 343-347 (1960)

3. B. Grünbaum, G.C. Shepard, Tilings and patterns: an introduction, W.H. Freeman and Co., New York, NY (1989)

4. M. De Graef, M.E. McHenry, Structure of materials, Cambridge University Press, Cambridge, UK (2008)

5. S. Baranidharan, E.S.R. Gopal, V. Sasisekharan, Generation of aperiodic tilings with fivefold symmetry by the method of interecting decogons and diffraction from finite size tilings, Pramana - J. Phys., 34(6) 537-553 (1990)

6. H. Masuda, H. Asoh, M. Wantanabe, K. Nishio, M. Nakao, T. Tamamura, Square and triangular nanohole array architectures in anodic alumina, Adv. Mater. 13 (3) 189-192 (2001)

7. K.S. Novoselov, D. Jiang, F. Schedin, T.J. Booth, V.V. Khotkevich, S.V. Morozov, A.K. Geim, Two-dimensional atomic crystals, Proc. Nat. Acad. Sci. 102 10341-10453 (2005)

8. J.C. Meyer, A.K. Geim, M.I. Katsnelson, K.S. Novoselov, T.J. Booth, S. Roth, The structure of suspended graphene sheets, Nature 446 60-63 (2007)

9. M. Krajci, J. Hafner, J. Ledieu, V. Fournée, R. McGrath, Quasiperiodic Pb monolayer on the fivefold $i$-Al-Pd-Mn surface: Structure and electronic properties, Phys. Rev. B 82 085417 (2010)

10. A. Vodopivec, F.H. Kaatz, B. Mohar, Topographical distance matrices for porous arrays, J. Math. Chem. 47 1145-1153 (2010)

11. E. Estrada, N. Hatano, Statistical-mechanical approach to subgraph centrality in complex networks, Chem. Phys. Lett., 439 247-251 (2007)

12. A. Clauset, C.R. Shalizi, M.E.J. Newman, Power-law distributions in empirical data, Siam Review, 51 661-703 (2009)

13. C.E. Shannon, A mathematical theory of communication, Bell Syst. Tech. J. 27 379423 (1948); 27 623-656 (1948)

14. N. Ikeda, Estimation of power-law exponent of degree distribution using mean vertex degree, Modern Physics Letters B, 23 (17) 2073-2088 (2009) 
Table 1

\begin{tabular}{|c|c|c|}
\hline Nearest Neighbor & 1 & 2 \\
\hline Triangle $a$ & 1 & $\sqrt{ } 3 \approx 1.732$ \\
\hline$L_{D}$ Triangle & 6 & 6 \\
\hline Hexagon $a$ & 1 & $\sqrt{3} \approx 1.732$ \\
\hline$L_{D}$ Hexagon & 3 & 6 \\
\hline Square $a$ & 1 & $\sqrt{ } 2 \approx 1.414$ \\
\hline$L_{D}$ Square & 4 & 4 \\
\hline
\end{tabular}

First and second neighbors for the regular tilings.

Table 2

\begin{tabular}{|c|c|c|c|c|c|}
\hline Graph & $a$ & $b$ & $c$ & $d_{\infty}$ & $R^{2}$ \\
\hline Random & 2 & $-1.013 \times 10^{-6}$ & 55.32 & 1.322 & 0.996 \\
\hline Hexagon & 9 & $-1.172 \times 10^{-11}$ & 28.73 & 3 & 0.979 \\
\hline Square & 9 & $-2.213 \times 10^{-10}$ & 20.5 & 4 & 0.984 \\
\hline Triangle & 14 & $-5.755 \times 10^{-11}$ & 17.02 & 6 & 0.984 \\
\hline Penrose & 65 & $-3.073 \times 10-8$ & 17.12 & 4 & 0.972 \\
\hline
\end{tabular}

Fit parameters for the degree distributions of Figure 5 (using equation 9).

\section{Figure 1}

Rhombs for 5 - fold Penrose tilings.

Figure 2

Plot of the number of vertices with their local degree for a Penrose tiling consisting of 12,079 total vertices.

Figure 3

MATLAB plots of:
(a) random graph
(b) hexagonal graph
(c) square graph
(d) triangular graph.
(e) Penrose tiling

\section{Figure 4}

(a) Plot of entropy / link vs. number of links.

(b) Plot of - enthalpy / link vs. number of links.

(c) Plot of - free energy / link vs. number of links.

\section{Figure 5}

Plot of the number of vertices vs. degree for each graph. The vertical lines are asymptotes to the fit. 
Figure 1
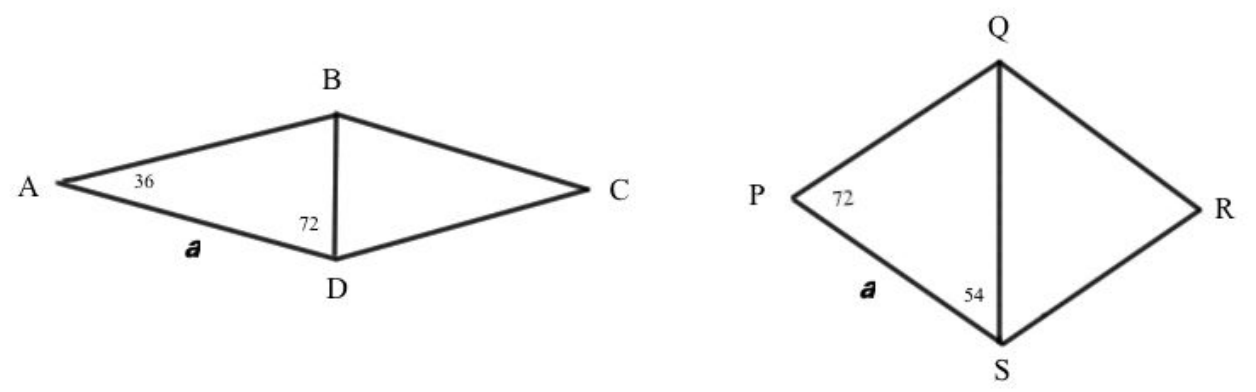

Figure 2

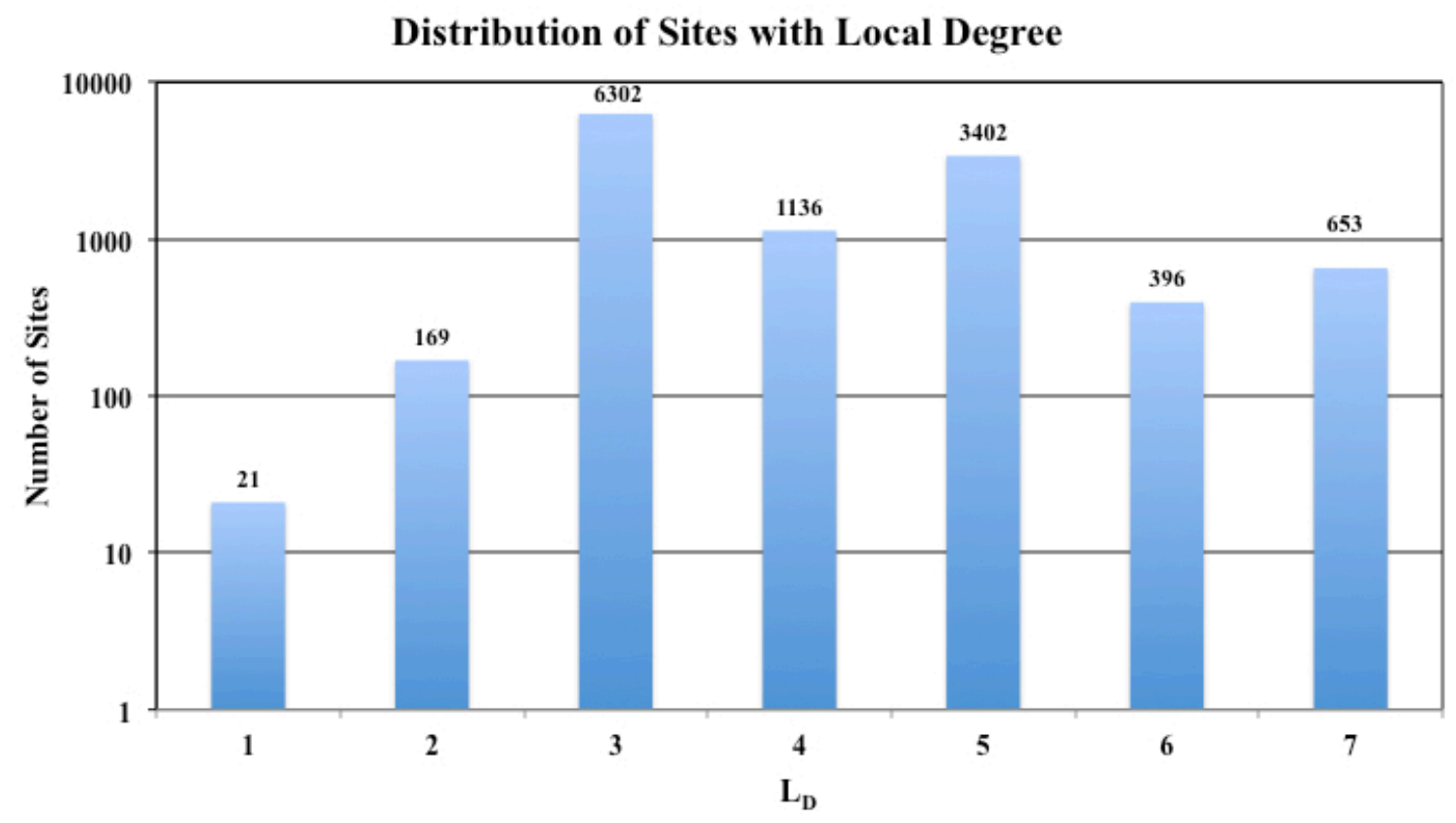


Figure 3(a)

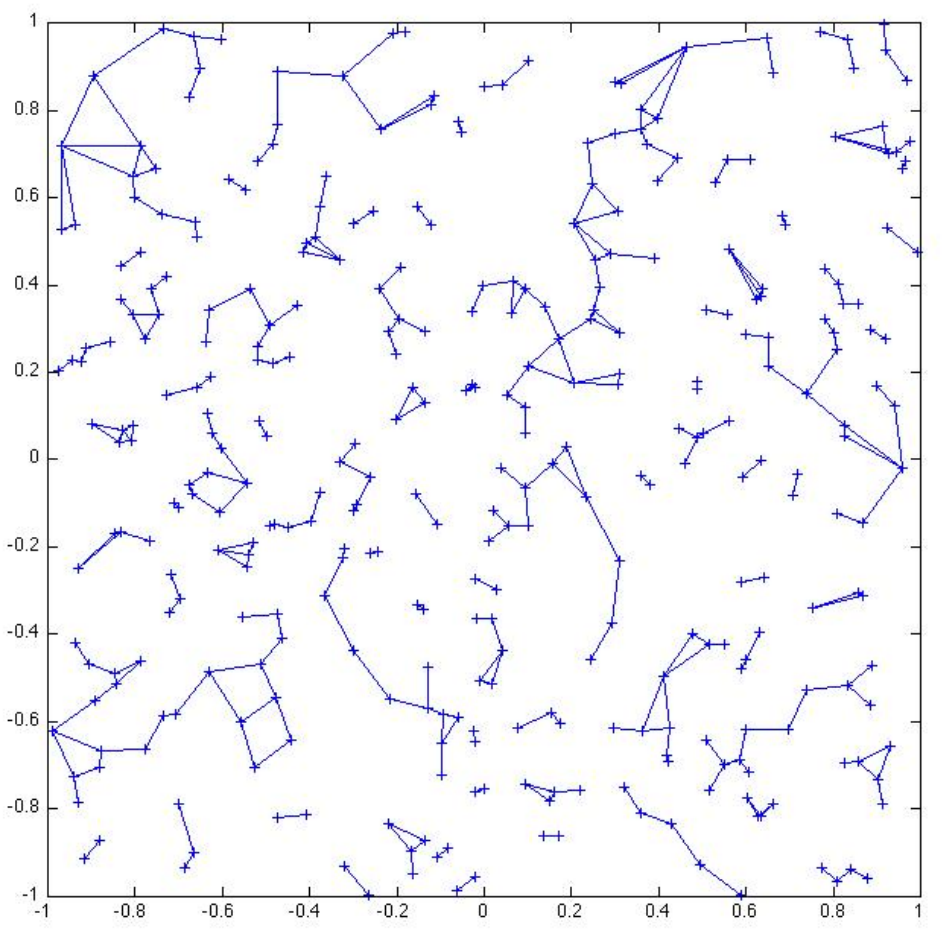

Figure 3(b)

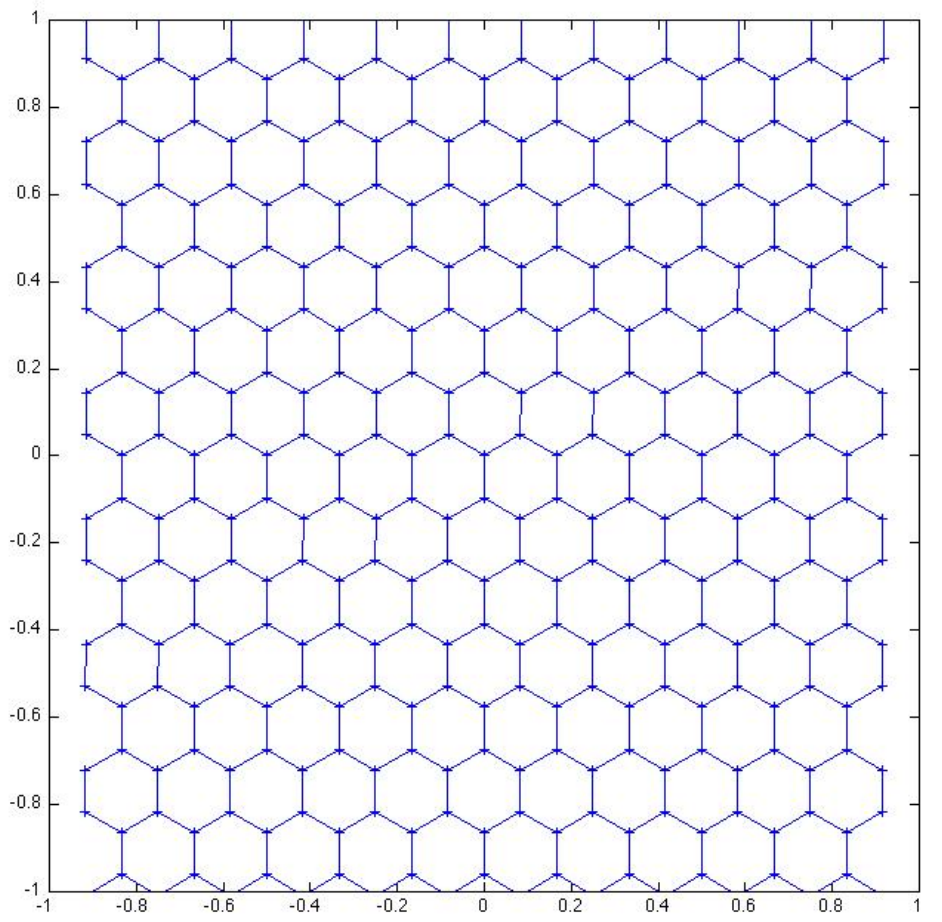

Figure 3(c) 


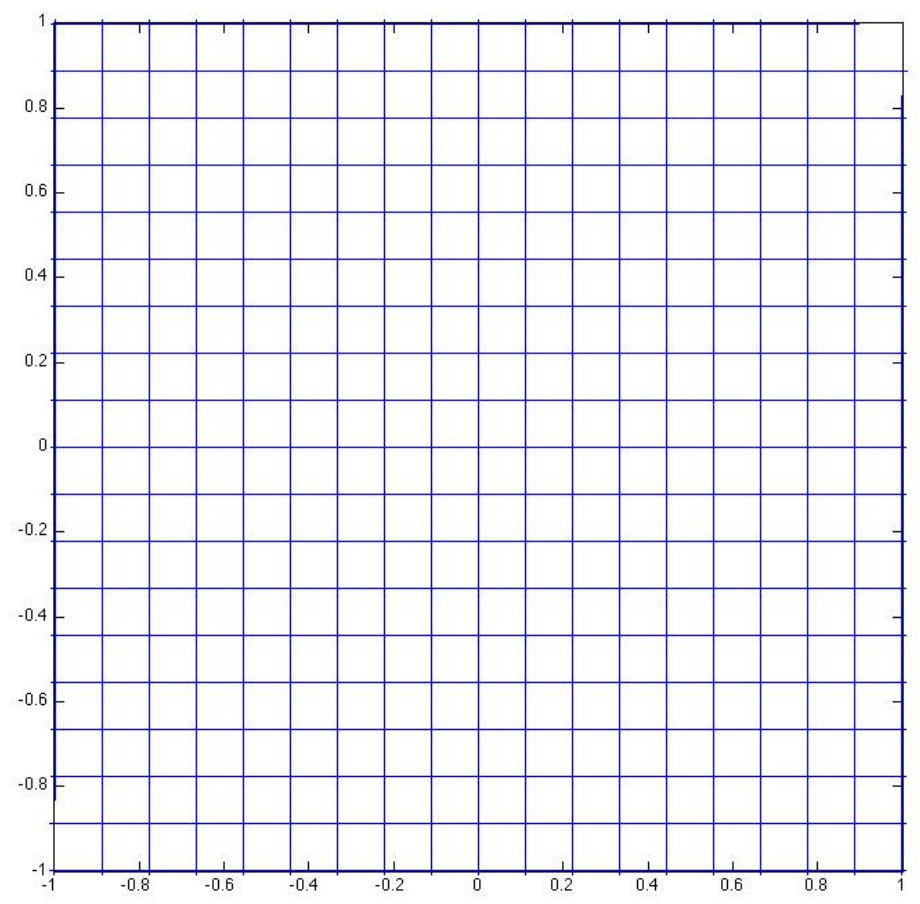

Figure 3(d)

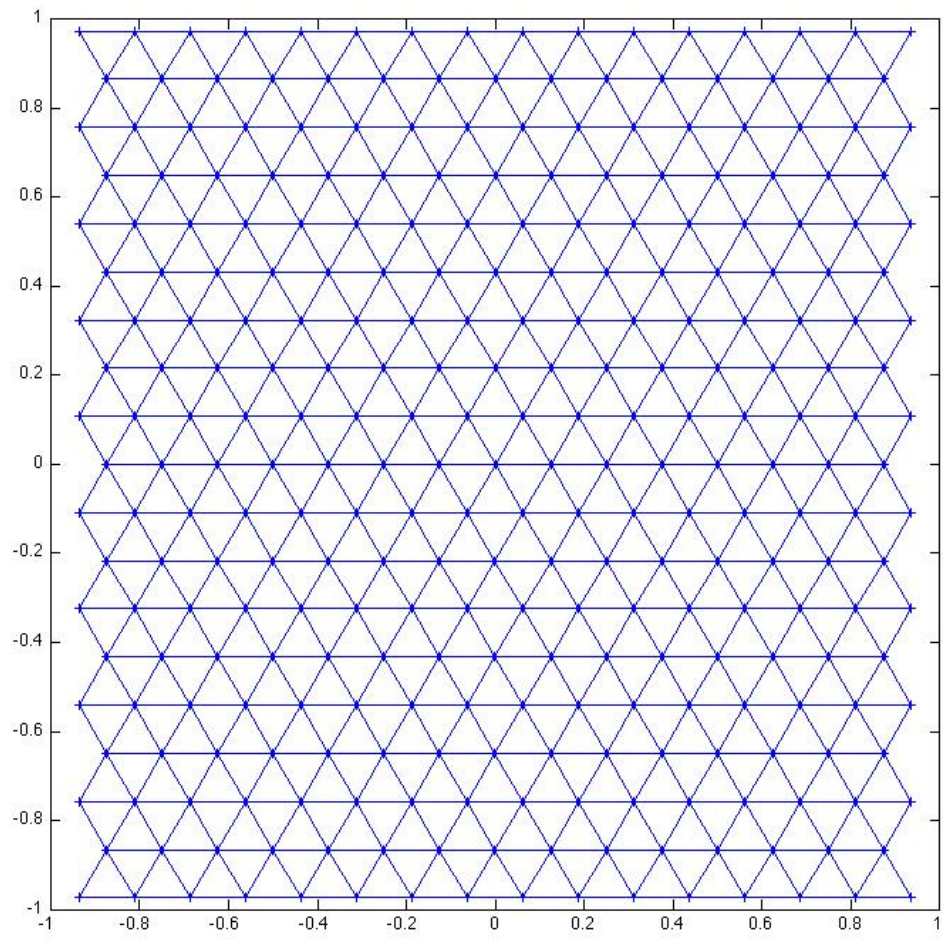

14 
Figure 3(e)

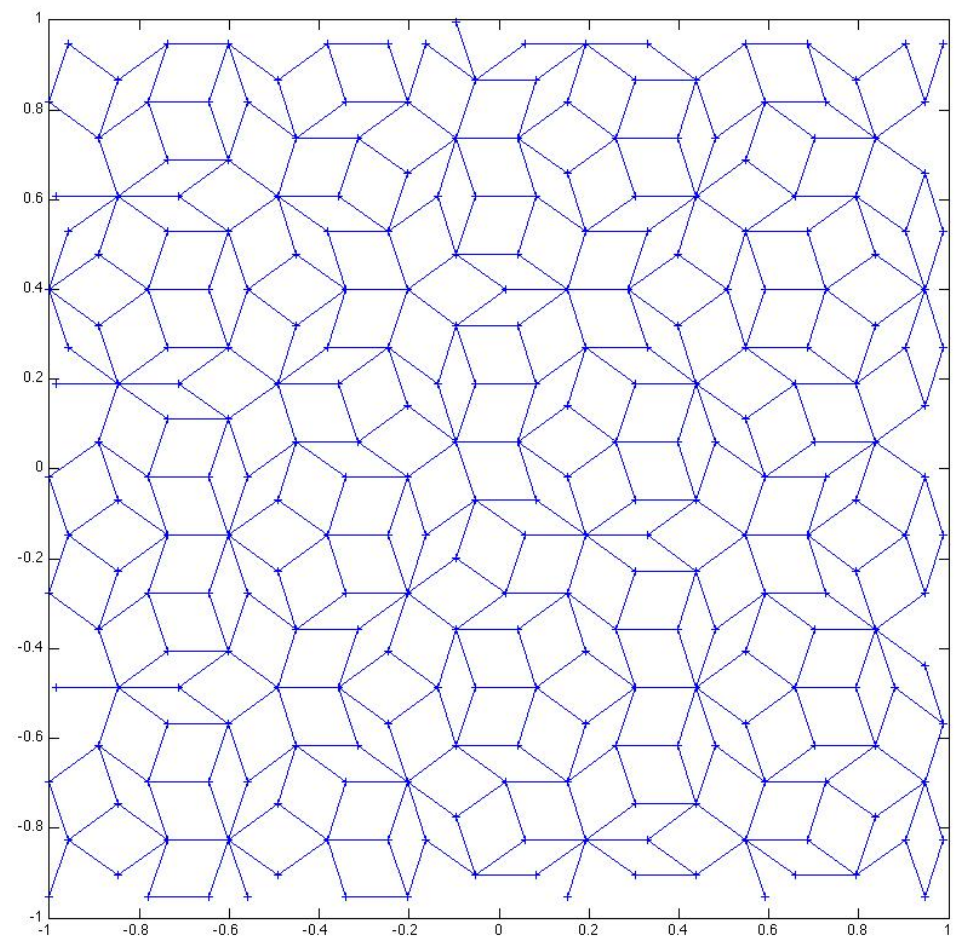

Figure 4(a)

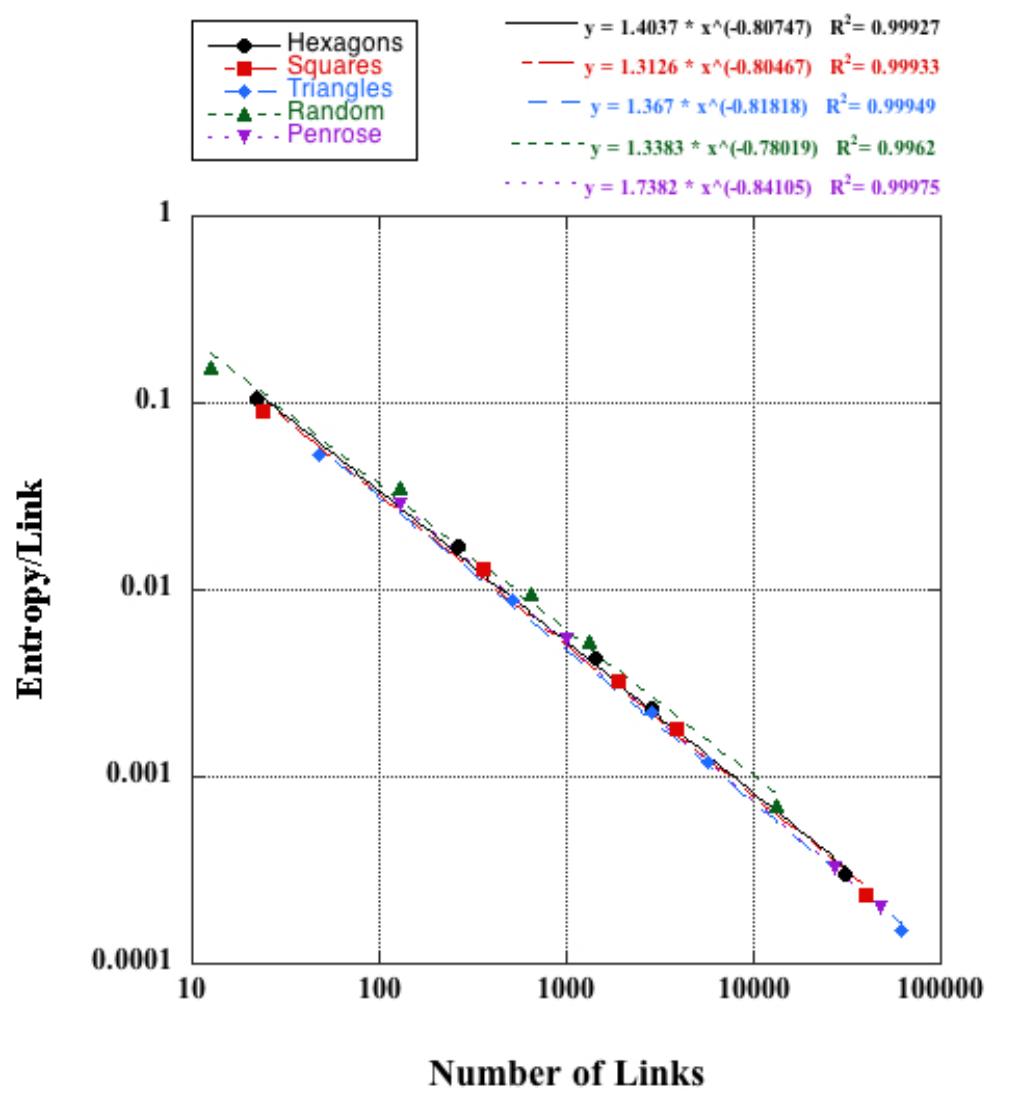


Figure 4(b)

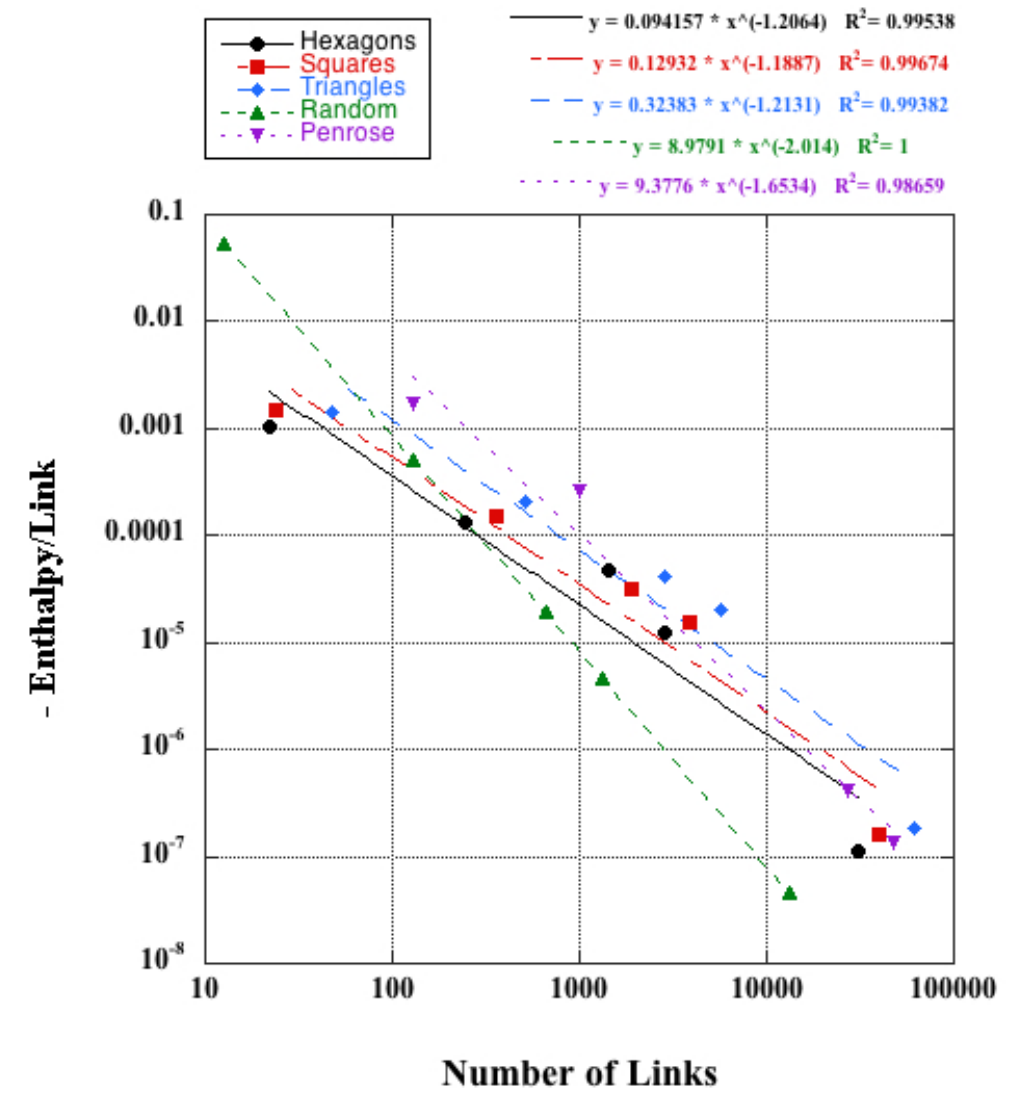

Figure 4(c)

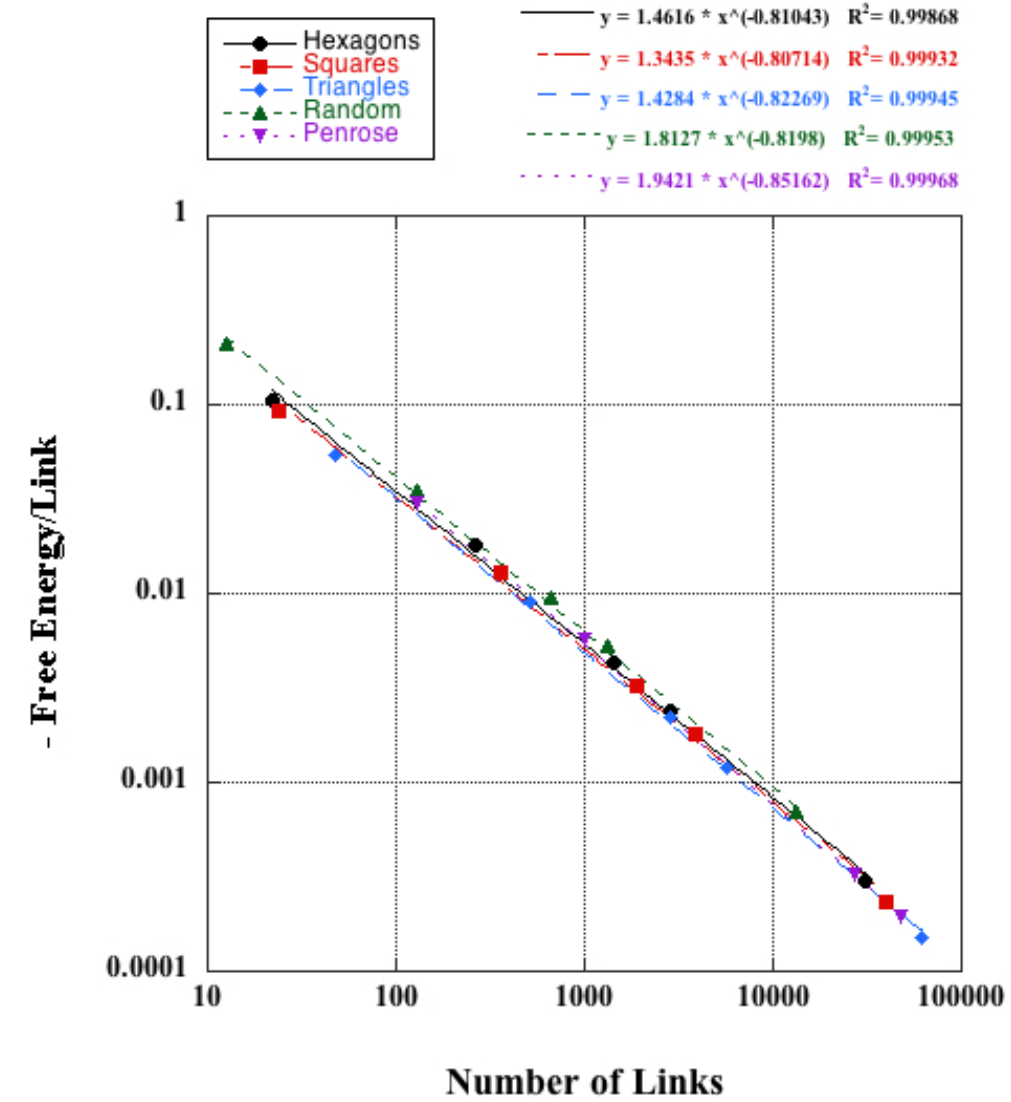


Figure 5

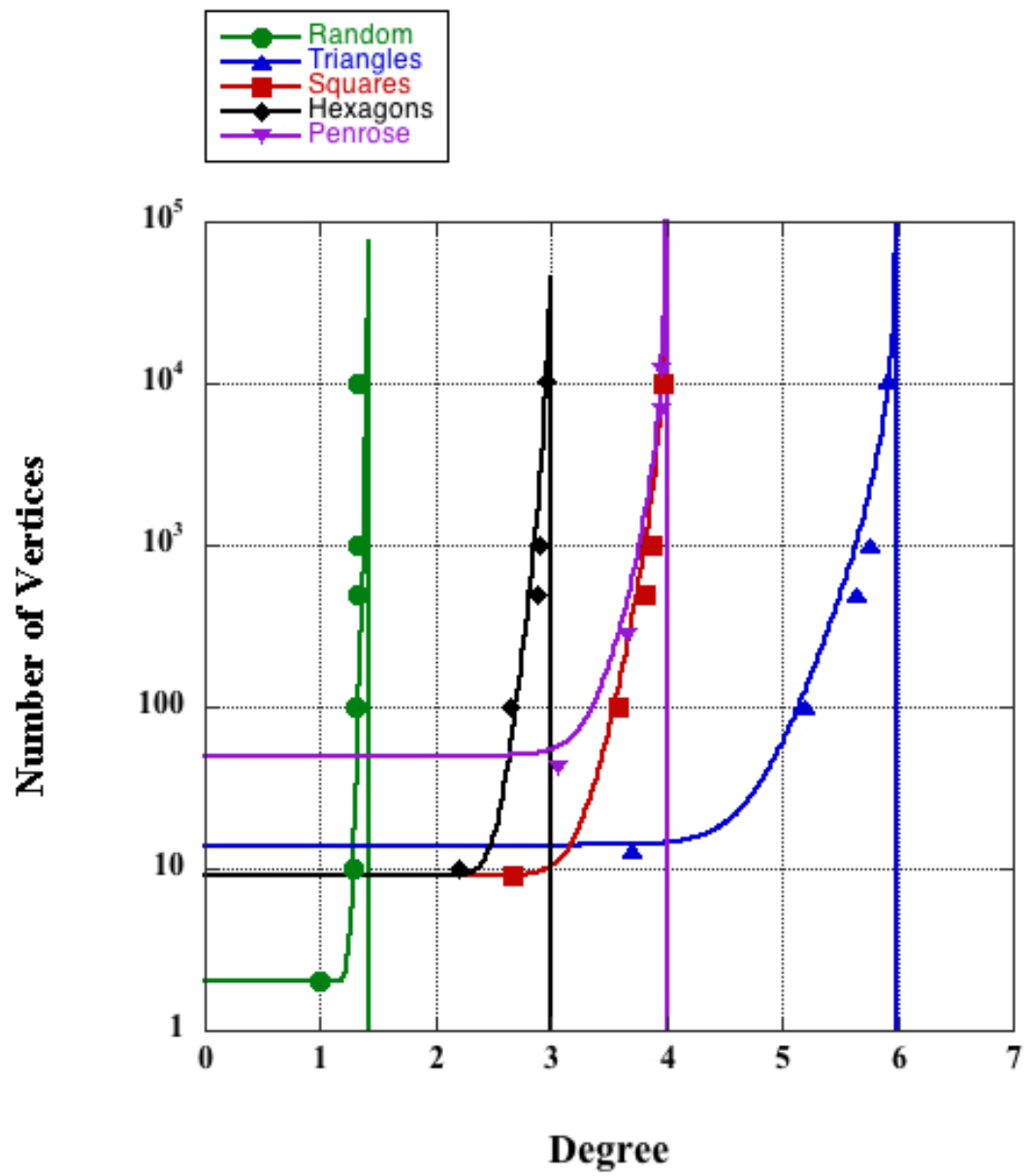

